\title{
Nanomolding Based Fabrication of Synthetic Gecko Foot-Hairs
}

\author{
Metin Sitti and Ronald S. Fearing \\ Dept. of EECS, University of California, Berkeley, CA 94720, USA \\ \{sitti,ronf\}@eecs.berkeley.edu
}

\begin{abstract}
This paper proposes two different nanomolding methods to fabricate synthetic gecko foot-hair nanostructures. The first method uses Atomic Force Microscope (AFM) probe indented flat wax surface and the second one uses a nano-pore membrane as a template. These templates are molded with silicone rubber, polyamide and polyester type of polymers under vacuum and the template is peeled off or etched away. Results show that these synthetic nano-hair prototypes have adhesion close to the predicted values for natural specimens (around 100 $\mathrm{nN}$ each).
\end{abstract}

\section{INTRODUCTION}

Geckos have attracted a great amount of attention from scientists for centuries due to their exceptional climbing capabilities on wet or dry and molecularly smooth or very rough surfaces. They have compliant and dry micro/nanoscale high aspect-ratio beta-keratin structures at their feet to adhere to any surface with a controlled contact area [1]. This adhesion is mainly due to the molecular forces such as van der Waals force [2]. Biological gecko hairs start from the micrometer scale (stalks) and go down to nanometer scale (spatular stalks) by branching [1]. Also, there are oriented caps (spatulae) at the very end of spatular stalks. This paper is focused on fabricating synthetic gecko foot-hair spatular stalks (nano-hairs) and setal stalks (micro-hairs). Nanomolding is utilized as the fabrication method, and two different techniques are proposed. At first, Atomic Force Microscope (AFM) probe based indenting is utilized to fabricate a master template, and this template is molded with silicone ruber and polyester. These synthetic nano-hair prototypes have adhesion close to the predicted values for natural specimens (around $100 \mathrm{nN}$ each). Next, nano-pore membranes fabricated by self-organization are used as master templates and are molded with polyamide and silicone rubber types of polymers to obtain high aspect ratio nano-hairs.

\section{SYNTHETIC HAIR DESIGN}

The basic design features of the synthetic hair fabrication can be given as follows: (1) High aspect ratio micrometer $(1: 10-30)$ and nanometer (1:20-50) scale structure fabrication with diameters of $3-10 \mu \mathrm{m}$ and $50-500 \mathrm{~nm}$ respectively, (2) Maximize micro/nano-hair density (number of hairs in a given area, e.g. $1 \mathrm{~cm}^{2}$ ) for higher adhesion, (3) Maximize nano-hair stiffness to prevent matting, (4) Material properties of synthetic hairs: Young's modulus of 0.1-20 GPa, hydrophobic, and high tensile strength.

\section{FABRICATION METHOD}

To fabricate synthetic hairs with above features, a master template that has micrometer- or nanometer-scale highaspect-ratio holes representing the negative of the synthetic hairs is molded with liquid polymers. Then the molded polymer is separated from the template by peeling off or etching. Two different methods using this nanomolding technique are explained below.

\section{III.1. Method I: Nanorobotic Imprinting}

For the first method, the shape of an existing master 1) single sharp probe such as AFM, STM, glass pipette, etc., 2) array of these probes, 3) any other high aspect ratio micro/nano-structure array is imprinted on a flat soft surface by indenting. Previously, nano-tip arrays are used as templates for imprint patterning of a polystyrene surface with $0.8 \mu \mathrm{m}$ diameter and $3 \mu \mathrm{m}$ depth holes [3]. Also, PMMA is indented by an AFM probe for datastorage applications [4] or by a micro-fabricated tip array and molded with a metal layer for patterning down to 40 nm metallic contacts [5].

In this paper, a single AFM probe (Nanosensors Inc., 42 $\mathrm{N} / \mathrm{m}$ stiffness, conical tip of apex radius $10-20 \mathrm{~nm}$, and 15 $\mu \mathrm{m}$ height) is used to indent a flat wax surface (J. Freeman Inc.). A single probe indenting can give the flexibility of even fabricating oriented hairs as can be seen in Fig. 1. By indenting the wax surface, the template as given in Fig. $2 \mathrm{a}$ was obtained. The profile of the indented wax surface can be seen in Fig. 2b. After molding with silicone rubber or any other polymer and separating the polymer from the wax template by peeling, synthetic nano-hairs of Fig. 2c were obtained. This process can be repeated autonomously to fabricate a large number of nano-hairs. An array of rubber nano-hairs made by manual step-andrepeat indenting is displayed in Fig. 3. 


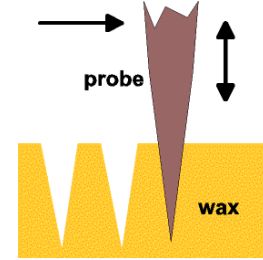

(a)

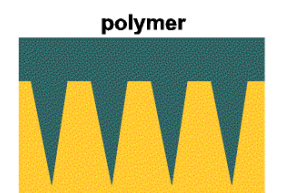

(b)

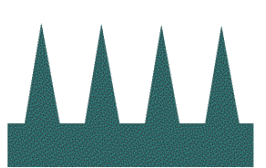

(c)
Fig. 1. Synthetic hair fabrication by the Method I: (a) Indenting a flat wax surface using a micro/nano-fabricated probe, (b) molding it with a polymer, (c) separating the polymer from the wax by peeling.

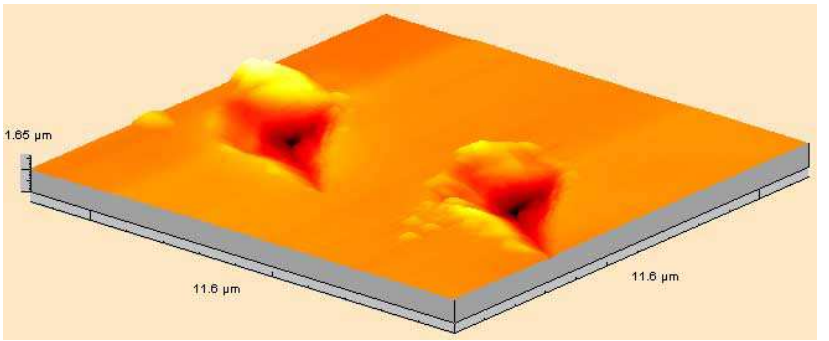

(a)

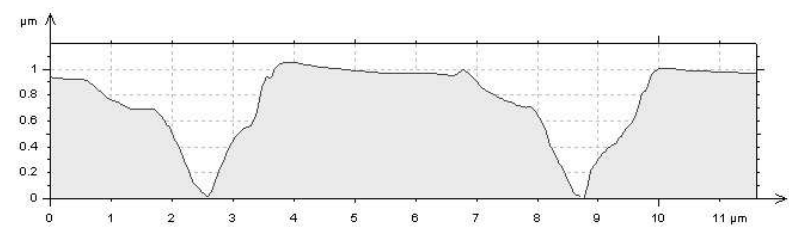

(b)

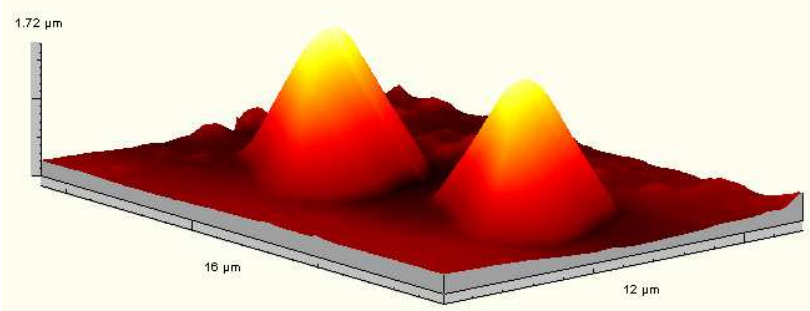

(c)

Fig. 2. 3D AFM tapping mode image of (a) the AFM probe based indented flat wax surface, (b) profile of the indented wax surface, (c) molded and peeled off silicone rubber nano-hairs.

Synthetic nano-hairs were fabricated from two different hydrophobic polymer materials (around $87^{\circ}$ contact angle with DI water), silicone rubber (Dow Corning Inc., HS II) and polyester resin (TAP Plastics Inc.). Silicone rubber Young's modulus was measured as $0.57 \mathrm{MPa}$. It was determined by measuring the stiffness of a molded knownsize rectangular rubber beam. However, feather betakeratin is measured as 1-10 GPa [6], and gecko foot-hairs is estimated in the range of $1-15 \mathrm{GPa}$ from our measurements using an AFM probe based bending of a single Tokay gecko stalk. While rubber has good adhesive properties, we had some concern that the natural stickiness of rubber might be contributing the measured adhesion force, rather than a more universal van der Waals attraction. Thus, polyester was also tried as the molding polymer material. Polyester Young's modulus is measured as around $0.85 \mathrm{GPa}$. Thus, it is a better match to the natural nano-hair modulus. A molded polyester bump is shown in Fig. 4.

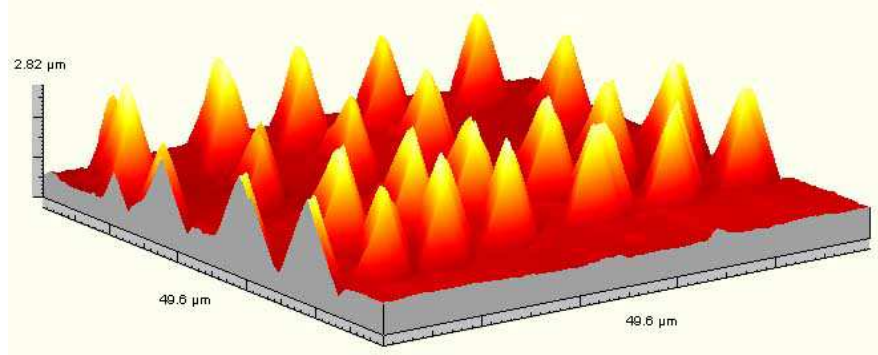

Fig. 3. 3D AFM tapping mode image of a silicone rubber nanohair array fabricated by repeated AFM probe indenting.

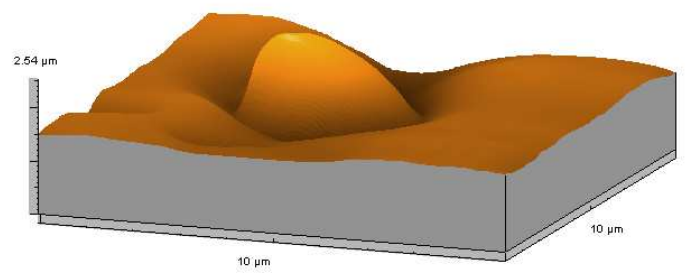

Fig. 4. A polyester synthetic nano-hair fabricated by molding an AFM probe indented wax surface.

To characterize the adhesion of synthetic hairs to a silicon surface with a natural oxide layer, pull-off force measurements were conducted using unloading forcedistance plots as in Fig. 5 with a tipless rectangular silicon AFM probe with $1.75 \mathrm{~N} / \mathrm{m}$ stiffness and $390 \mathrm{~nm} / \mathrm{s}$ retraction speed. In the unloading force-distance curves, the maximum attractive (pull-off) force gives the adhesion of the synthetic nano-hair to the probe $\mathrm{SiO}_{2}$ surface. Measurements were made in a laboratory environment with $25^{\circ} \mathrm{C}$ and $58 \%$ relative humidity. Pull-off force was measured as $181 \pm 9 \mathrm{nN}$ (25 samples) for silicone rubber nano-hair with tip radius range of 230-440 nm (measured from the 3D AFM image) and $294 \pm 21 \mathrm{nN}$ (20 samples) for polyester nano-hair with tip radius of $350 \mathrm{~nm}$. We thus have shown good repeatability. The RMS surface roughness of silicone rubber and polyester flat substrates are measured by AFM as around $3 \mathrm{~nm}$ and $5 \mathrm{~nm}$ respectively. Since these values are relatively small and we could not measure the roughness at the tip of the synthetic nano-hairs directly, the roughness effect on surface forces was neglected.

Pull-off force is given by the JKR theory as $\mathrm{F}=1.5 \pi \mathrm{RW}$, where $W \approx 2 \sqrt{ } \gamma_{1} \gamma_{2}$ [7]. $\gamma_{1}=160 \pm 40 \mathrm{~mJ} / \mathrm{m}^{2}$ [8] and $\gamma_{2}$ 
are the surface energies of the $\mathrm{SiO}_{2}$ layer and the polymer respectively. For silicone rubber with $\gamma_{2}=21.4 \mathrm{~mJ} / \mathrm{m}^{2}$ [9], $185 \mathrm{nN}$ pull-off force is theoretically expected taking $\mathrm{R} \approx 335 \mathrm{~nm}$. For polyester with $\gamma_{2}=44.6 \mathrm{~mJ} / \mathrm{m}^{2}$ [9], $279 \mathrm{nN}$ is expected. These expected adhesion forces are very close to the measured ones.

This adhesive force is consistent with van der Waals force. For calculating the van der Waals contribution of these adhesion forces, $\mathrm{F}_{\mathrm{vdw}}=\mathrm{HR} / 6 \mathrm{~d}_{0}{ }^{2}$ is used where $\mathrm{d}_{0} \approx 0.165 \mathrm{~nm}$ is the approximate interfacial cut-off distance [7] and $\mathrm{H}$ is the Hamaker constant. Using $\mathrm{H} \approx 45 \times 10^{-21} \mathrm{~J}$ (estimated from $\mathrm{H}=2.1 \times 10^{-24} \gamma$ [7]) for rubber, $\mathrm{H}=60.5 \times 10^{-21} \mathrm{~J}[10]$ for polyester, and $\mathrm{H}=68.5 \times 10^{-21} \mathrm{~J}[10]$ for $\mathrm{SiO}_{2}, \mathrm{~F}_{\mathrm{vdw}}=114$ $\mathrm{nN}$ and $\mathrm{F}_{\mathrm{vdw}}=139 \mathrm{nN}$ are predicted for silicone rubber and polyester respectively. Thus, $47-63 \%$ of the adhesion forces of the synthetic nano-hairs are consistent with the van der Waals forces while the rest could be due to polar interactions, other adhesive effects and surface roughness effects.

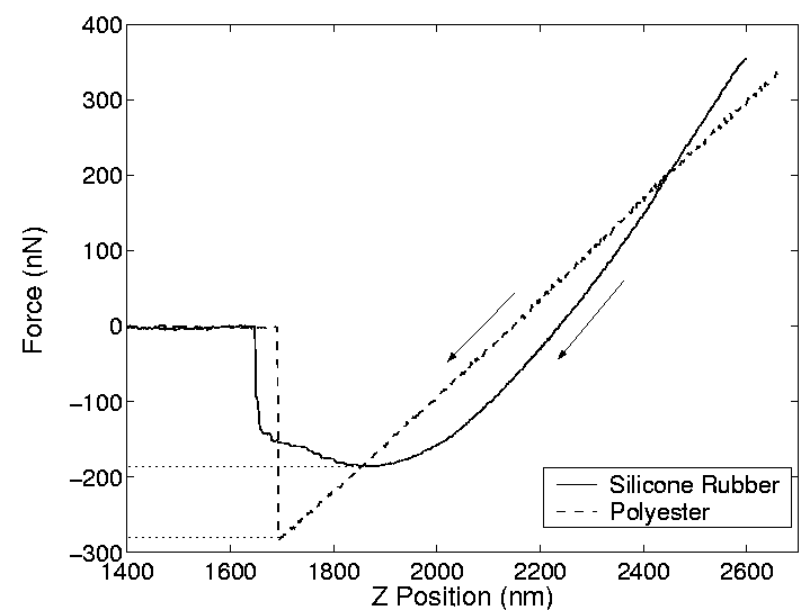

Fig. 5. Pull-off force measurements of the synthetic (silicone rubber (dashed line) and polyester (solid line)) nano-hairs using a tipless AFM probe.

\section{III.2. Method II: Parallel Fabrication}

For the second method, a membrane with self-organized high aspect ratio pores is used as the master template and molded with a liquid polymer. Two types of membranes were used: alumina (Nanopore, Whatman Inc.) and polycarbonate (Poretics, Osmonics' Lab Inc) membranes. They have different range of diameter, density and thickness of pores, Young's modulus, etching properties, etc. (Table 1).

\begin{tabular}{|l|l|l|l|}
\hline Material & $\begin{array}{l}\text { Pore Diameter } \\
(\mu \mathrm{m})\end{array}$ & $\begin{array}{l}\text { Thickness } \\
(\mu \mathrm{m})\end{array}$ & $\begin{array}{l}\text { Pore Density } \\
\left(\text { pores } / \mathrm{cm}^{2}\right)\end{array}$ \\
\hline Alumina & $0.02-0.2$ & 60 & $10^{9}$ \\
\hline Polycarbonate & $0.02-20$ & $7-14$ & $10^{5}-10^{8}$ \\
\hline
\end{tabular}

Table 1. Properties of nano-pore membranes.
First, an alumina membrane with $200 \mathrm{~nm}$ diameter and 60 $\mu \mathrm{m}$ thick perpendicular nano-pores was molded with polyamide (PI-2525, HD Microsystems Inc) and silicone rubber (HSII) under vacuum. After molding, polyamide was cured at $100^{\circ} \mathrm{C}$ for $1 \mathrm{hr}, 200^{\circ} \mathrm{C}$ for $30 \mathrm{~min}$, and $350^{\circ} \mathrm{C}$ for $1 \mathrm{hr}$, and the alumina membrane is etched away by $\mathrm{HCl}$ in $2 \mathrm{hr}$. Scanning Electron Microscope (SEM) micrographs of the alumina membrane top-view and polyamide nano-hairs are given in Fig. $6 \mathrm{a}$ and $6 \mathrm{~b}$ respectively. Here, membrane and the polyamide hairs are coated with a $10 \mathrm{~nm}$ thick gold layer for SEM imaging. Since wet etching is utilized and nano-hairs are extremely compliant (long), hydrophobic polyamide hairs stuck to each other. Moreover, silicone rubber was also molded using the same membrane and the resulting nano-hairs are shown in Fig. 6c. As can be seen from the results, $60 \mu \mathrm{m}$ long nano-hairs are too compliant and too dense (i.e. too close to each other) to prevent self-sticking while molding of down to $200 \mathrm{~nm}$ nano-pores with liquid polymers under vacuum is demonstrated successfully. Therefore, synthetic hairs should be much shorter and less dense, and/or external excitation such as ultrasonic vibration or proper surfactants for the given etchant should be used during the wet etching process to minimize the selfsticking problem due to hydrophobic attraction.

For shortening the nano-hairs and decreasing the nano-hair density, polycarbonate membranes were used. These membranes have a random orientation of the nanopores $\left( \pm 15^{\circ}\right)$ created by a nuclear track etch. The SEM micrograph of a polycarbonate membrane is displayed in Fig. 7a. A $8 \mu \mathrm{m}$ diameter polycarbonate membrane was molded with silicone rubber under vacuum and the rubber is peeled off from the membrane after curing at room temperature for $24 \mathrm{hr}$. Resulting rubber hairs with around $6 \mu \mathrm{m}$ diameter and length are shown in Fig. 7b. Smaller diameter (down to $200 \mathrm{~nm}$ ) membrane molding is currently in progress. Adhesion of rubber hair array in Fig. $7 \mathrm{~b}$ to a flat glass substrate is measured using a force sensor as around $2.8 \mathrm{mN} / \mathrm{cm}^{2}$ for a $25 \mathrm{mN}$ preload. This implies around $60 \mathrm{nN}$ adhesion for each single hair using the fabricated hair density of $5 \times 10^{4}$ pores $/ \mathrm{cm}^{2}$ and assuming all hairs contact to the substrate.

\section{CONCLUSION}

Synthetic gecko foot-hair nano-hairs were fabricated by two different nanomolding techniques. AFM probe-based indented flat wax surface in the Method I and selforganized alumina and polycarbonate nano-pore membranes in the Method II were used as the master templates. The Method I resulted in nano-hairs with adhesion forces in the range of $180-300 \mathrm{nN}$ while the 
biological nano-hairs give around 50-300 $\mathrm{nN}$ [1]. Thus, the generated sticking forces are similar, and we have shown that the adhesive forces are 1) relatively independent of material properties, 2) repeatable, 3) consistent with a standard adhesive model. The Method II preliminary results are promising for mass production, but further optimization is required to obtain stalks with appropriate length and diameter.

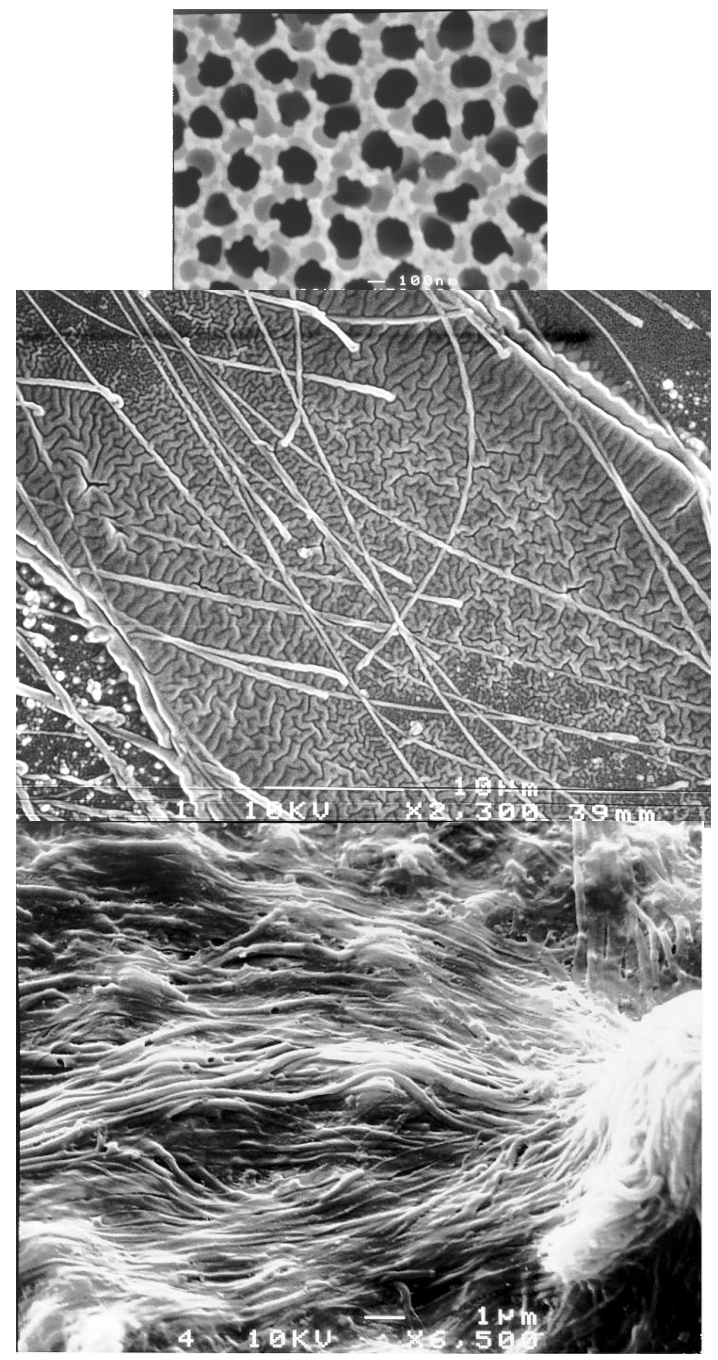

Fig. 6. SEM micrographs of (a) porous alumina membrane topview (uppermost), (b) molded polyamide nano-hairs (middle), (c) molded silicone rubber nano-hairs (lowermost).

\section{ACKNOWLEDGEMENTS}

This work was supported by DARPA N66001-00-C-8047 and N66001-01-C-8072 from the Controlled Biological and Biomimetic Systems Program, Defense Sciences Office, under the auspices of Dr. Alan Rudolph. Authors would thank Prof. Kellar Autumn (Lewis and Clark College) for measuring adhesion of the rubber hair array, and Murat Ozturk for assisting in membrane molding experiments.

\section{REFERENCES}

[1] K. Autumn, and et. al, "Adhesive force of a single gecko foot-hair," Nature, vol. 405, pp. 681-685, June 82000.

[2] K. Autumn, M. Sitti, Y. Liang, and et. al, "Evidence for van der Waals attachment by gecko foot-hairs inspires design of synthetic adhesive," Proc. of the National Academy of Science, 2002 (under review).

[3] T. Dam and P. Pantano, "Nanotip array photoimprint lithography," Review of Scientific Instruments, vol. 70, no. 10, pp. 3982-6, Oct. 1999.

[4] U. Durig and et al., "Millipede-an AFM data storage system at the frontier of nanotribology," Tribology Letters, vol. 9, pp. 25-32, 2000.

[5] T. Borzenko and et al., "Imprint with sharp tip stamps," Microelectronic Engineering\}, vol. 57-58, pp. 389-396, 2001.

[6] J. Vincent, Structural Biomaterials, rev. ed., Princeton Univ. Press, 1990.

[7] J. Israelachvili, Intermolecular and Surface Forces, 2nd Ed., Academic Press, London, 1992.

[8] M. Yu, T. Kowalewski, and R. Ruoff, "'Structural analysis of collapsed and twisted multi-walled carbon nanotubes by Atomic Force Microscopy,” Phys. Rev. Lett., vol. 86, 2000.

[9] R. French, "Origins and applications of London dispersion forces and Hamaker constants in ceramics," J. Am. Ceram. Soc., vol. 83, pp. 2127-46, 2000.

[10] L. Li, V. Mangipudi, M. Tirrell, and A. Pocius, "Direct measurement of surface and interfacial energies of glassy polymers and PDMS," Fundamentals of Tribology and Bridging the Gap between the Macro-and Micro/Nanoscales, Kluwer Academic Pub., Netherlands, 2001.

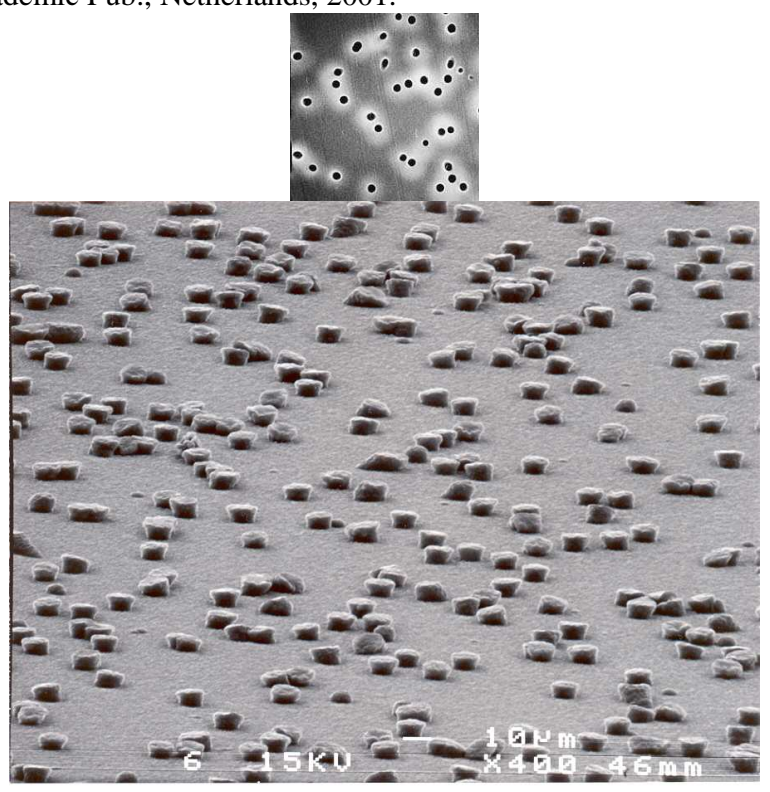

Fig. 7. SEM micrographs of (a) polycarbonate membrane topview image (upper), and (b) molded silicone rubber hairs with around $6 \mu \mathrm{m}$ diameter, $6 \mu \mathrm{m}$ length and $5 \times 10^{4}$ pores $/ \mathrm{cm}^{2}$ density (lower). 